\title{
Ten years of Methods
}

The decade since the launch of Nature Methods has been one of intense and dynamic development in biological research methods. We predict this will continue.

Since the first issue of Nature Methods was published in October 2004, the journal's goal has been to provide a high-visibility, high-quality, interdisciplinary forum for the publication of new and improved research methods. Though nobody questioned the importance of methodology for biological progress, the publication landscape for methods papers at the time was bleak. As our first editorial noted, "Technical creativity is not always rewarded for its own, significant value." Our raison dêtre has been to change that.

It is our hope that in some measure we have succeeded, at least as gauged by the vibrancy of the journal, the accelerating pace and rising quality of submissions, the number of citations our papers receive, and the interest and support of scientists-you, our readers, referees and authors-in many research communities.

The biological scene of today is very different from what it was in 2004, in large part owing to methodological and technological leaps. Ten years ago, for instance, we had in hand a single human reference genome, the product of intense efforts using Sanger technology; now, with high-throughput sequencing, this number has risen into the thousands and will increase further.

To use a completely different example, in 2004 super-resolution microscopy was in its infancy and light-sheet microscopy had only just been applied to a developing embryo. Progress in instrumentation and analysis, not to mention the increasing excitement of biologists about imaging sharp, fast and deep, have since catapulted these approaches to the forefront of cell and developmental biology and neuroscience.

Ten years ago, the best proteomics groups, with supreme effort, could profile 1,000-2,000 proteins in a sample using mass spectrometry. Today, near-complete proteomes-including many post-translational modifications - can be not only identified, but also quantified, with substantially less work and with wellestablished metrics for quality control.

Neuroscience, meanwhile, has witnessed the rise of optogenetics within the past decade. The notion of using light to modulate neuronal activity was not new in 2004, but the application of the genetically encoded light-sensitive protein channelrhodopsin has since triggered the development of an arsenal of tools to activate and inhibit neuronal function.
Or, looking back at stem cell biology, ten years ago it had yet to be announced that somatic cells could be reprogrammed to 'induced' pluripotency. Now we have a new appreciation of the plasticity of cell fate, and research on many human cell types with a desired genetic background is within reach. And while the foundations for targeted genetic modification were laid over many decades, the remarkable ability afforded by designer nucleases, particularly the RNA-guided CRISPR-Cas9 system, to flexibly edit endogenous genes in cells and in organisms is of recent vintage.

The list could go on. In this tenth-anniversary issue, we have highlighted the ten areas of methods development that we think have had the biggest impact on biological research over the last decade (p1000-1001). Some of these-their history, their power and what is still needed for them to reach their full potential-are discussed in Commentaries in the issue (p1003-1020). On Methagora, you can look back with us over our first decade and find papers we have published in some of these areas.

Our 'top ten' choices are necessarily incomplete and subjective. Systems biology approaches to generate, integrate and interpret large data sets have changed life science research immeasurably, for instance. Separately, methods to culture biological material ex vivo by harnessing engineered environments or cellular self-organization are on the rise. Development in these areas, among others, is sure to continue.

A question that arose at the time of Nature Methods' launch was whether a journal devoted to methods development would have staying power. Might such a publication not write itself out of existence? But although development in some areas does slow down, many fields-including, notably, every one of our 'top ten'-are only accelerating. The rise of new technologies can enliven methods development even in wellestablished fields, as with the application of X-ray free-electron lasers to structural biology. And even as methods mature, their creative application to new problems and improvements to extend their reach and accessibility remain important for biologists.

Our view, ten years in, is that methods development will continue to drive biological research for decades to come. 